\title{
METHODS OF EXTRACTING ELECTRON-POSITRON ENHANCEMENT FACTORS FROM EXPERIMENTAL ACAR DATA
}

\author{
G. Kontrym-SzNAJD AND A. RUBASZEK \\ W. Trzebiatowski Institute of Low Temperature and Structure Research \\ Polish Academy of Sciences \\ P.O. Box 937, 50-959 Wrocław 2, Poland
}

\begin{abstract}
Methods of extracting the full shape of the electron-positron momentum densily from experimental angular correlation of positron annihilation radiation curves are discussed. The proposed analysis of experimental data allows more reliable verification of the form of electron-positron enhancement factors near the Fermi surface in simple metals.
\end{abstract}

PACS numbers: 78.70.Bj, 71.20.-b

\section{Introduction}

In the interpretation of experimental angular correlation of positron annihilation radiation (ACAR) data with respect to the electronic structure of materials, the knowledge of the electron-positron momentum dependent enhancement factors $\varepsilon(p)$ is necessary. In Ref. [1] we discuss the possibility of describing electron-positron correlation effects in simple metals within the model of jellium of some effective density. The applicability of electron gas theory to nearly-free electronic (NFE) populations (e.g., valence electrons in simple metals) has an essential significance. In calculations of positron annihilation characteristics in real metals the local density approach (LDA) has been recently quite often applied [2]. Nevertheless, there is a question which of alternative electron gas theories (for review see, e.g. [3]) provides correct enhancement factors $\varepsilon_{\text {jell }}\left(p, r_{\mathrm{s}}\right)$, where $r_{\mathrm{s}}$ is the electron density parameter. Before applying the jellium results to LDA calculations in real metals, we should verify $\varepsilon_{\text {jell }}\left(p, r_{\mathrm{s}}\right)$ comparing them with experimental data.

The main problem, however, connected with "experimental" enhancernent factors $\varepsilon_{\exp }(p)$ follows from the fact that the biparabolic approximation of $\varepsilon(p)$, applied to fitting of experimental ACAR data, is not valid even in the electron gas [4]. That is why the extraction of real momentum dependence of $\varepsilon_{\exp }(p)$ is of vital importance. This paper is devoted to the methods of elaboration of experimental positron annihilation data with respect to this problem. 


\section{Study of the enhancement factor from experimental ACAR data}

In the interpretation of experimental one-dimensional (1D) or two- dimensional (2D) ACAR data

$$
N\left(p_{z}\right)=\int \mathrm{d} p_{x} \mathrm{~d} p_{y} \rho(\boldsymbol{p}) \quad \text { or } \quad N\left(p_{x}, p_{y}\right)=\int \mathrm{d} p_{z} \rho(\boldsymbol{p}),
$$

electron-positron enhancement factors are usually approximated according to the biparabolic formula [5]:

$$
\varepsilon_{\mathrm{b}}\left(p / p_{\mathrm{F}}\right)=a+b\left(p / p_{\mathrm{F}}\right)^{2}+c\left(p / p_{\mathrm{F}}\right)^{4}
$$

where $p_{F}$ is the Fermi momentum. $\rho(p)$ in Eq. (1) denotes the momentum density of annihilation quanta and the ACAR spectra are normalized to corresponding projections of $\rho(\boldsymbol{p})$. However, as pointed out by Rubaszek and Stachowiak [4], biparabolic approximation (2a) is valid only for momenta $|p| \leq 0.8 p_{F}$, i.e. for about $50 \%$ of all electronic states. The ratio $(b+c) / a$ (which is more reliable than $b / a$ and $c / a$ separately [6]) cannot be identified with relative enhancement factor at the Fermi surface (FS), $\left[\varepsilon\left(p_{\mathrm{F}}\right)-\varepsilon(0)\right] / \varepsilon(0)$. A great caution should be therefore paid when conclusions about the parameters $b / a$ and $c / a$ are drawn from experimental data.

In majority of experimental works the coefficients $b / a$ and $c / a$ are determined either from densities $\rho_{\text {rec }}(p)$ reconstructed from experimental data (cf. Sec. 3), or from ACAR (1D or 2D) spectra. Dependently on the way of dealing with experimental data, however, the resulting values of the parameters $b / a$ and $c / a$ (as well as their sum) differ (Ref. [1] and discussion in Ref. [6]). This disagreement between "experimental" enhancement factors may be attributed to the fact that the biparabolic approximation (2a) is not valid for momenta close to the Fermi momentum. As a result, experimental values of $b / a, c / a$, and $(b+c) / a$ depend strongly on the choice of the interpolation points $\left(p_{1}, p_{2}, p_{3}\right)$. Moreover, they differ essentially depending on the way of dealing with the data (fitting to reconstructed density $\rho_{\text {rec }}(p)$ or to ACAR data). This fact is illustrated in Figs. 1 and 2. In Figs. 1a-c the relative differences between $\varepsilon(p)=\varepsilon_{\text {jell }}\left(p, r_{\mathrm{s}}=2\right)$ and its approximation $\varepsilon_{\mathrm{b}}(p)$ in the form (2a), where the parameters $b / a$ and $c / a$ were fitted to $\varepsilon(p)$ for three various sets of interpolation points, are displayed by dashed lines. The solid lines in Figs. 1a-c represent corresponding fits of $N_{\mathrm{b}}\left(p_{z}\right)$ (Eq. (3b)) to $N\left(p_{z}\right)$. In Fig. 2 the results obtained from $2 \mathrm{D}$ ACAR experimental data for $\mathrm{Na}$ and $\mathrm{K}$ are presented. The reconstructed densities, $\rho_{\text {rec }}(p)$, in these metals are based on the one $2 \mathrm{D}$ projection only. It is visible that in both cases for momenta up to $p \approx 0.75 p_{\mathrm{F}}$ the reconstruction method (fitting to $\rho_{\text {rec }}(p)$ ) leads to considerably smaller difference between the starting "true" enhancement factor and the fitted one than fitting parameters to 1D or 2D curves. However, when $p$ approaches the FS, $N\left(p_{z}\right)$ or $N\left(p_{x}, p_{y}\right)$ provide much better fit. The results presented above lead to the conclusion that simultaneous study of reconstructed densities and ACAR curves could help us to answer the question about the form of the enhancement factors in real metals. Since biparabolic formula (2a) does not describe properly $\varepsilon(p)$ near the FS, we propose to correct it in order to reproduce "true" momentum dependence of $\varepsilon(p)$. The corresponding enhancement factor may be written in the form [1]:

$$
\varepsilon(p)=\varepsilon_{\mathrm{b}}(p)+\varepsilon_{\mathrm{c}}(p) \Theta\left(0.8 p_{\mathrm{F}}-p\right),
$$




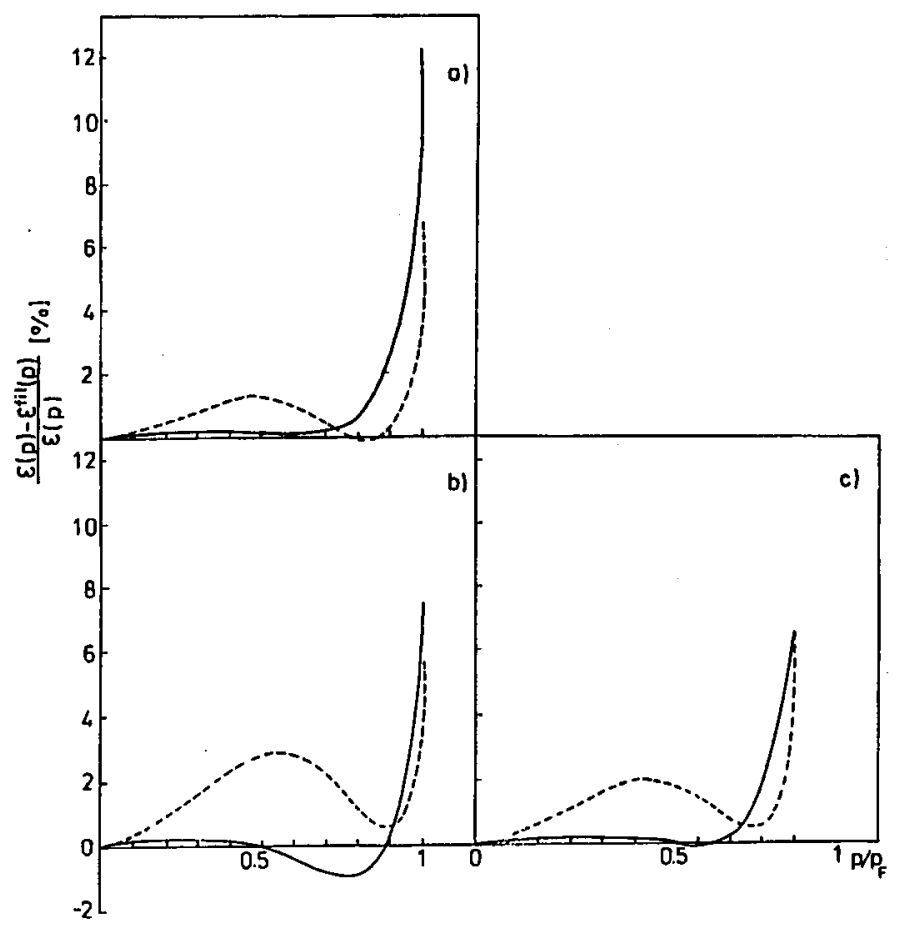

Fig. 1. Relative differences between values of $\varepsilon(p)=\varepsilon_{\text {jell }}\left(p, r_{\mathrm{s}}=2\right)$ and its biparabolic form $\varepsilon^{\text {fit }}(p)=\varepsilon_{\mathrm{b}}(p)$ for various sets of interpolation points $\left(p_{1}, p_{2}, p_{3}\right):(\mathrm{a})(0.1,0.5,0.7)$; (b) $(0,0.5,0.9)$; and (c) $(0.2,0.5,0.8)$. Solid and dashed lines describe the cases when the parameters $b / a$ and $c / a$ were fitted to $N\left(p_{z}\right)$ and $\varepsilon(p)$, respectively.

where $\varepsilon_{\mathrm{b}}(p)$ is given by formula $(2 \mathrm{a}), \Theta$ is the unit-step function and $\varepsilon_{\mathrm{c}}(p)$ is a correction function. If the enhancement factors $\varepsilon_{\text {jell }}\left(p, r_{\mathbf{s}}\right)$ following from the Bethe-Goldstone-type equation are considered, the function $\varepsilon_{\mathrm{c}}(p)$ may be approximated by the form

$$
\varepsilon_{c}\left(p / p_{\mathrm{F}}\right)=d /\left[1.06-\left(p / p_{\mathbf{F}}\right)^{2}\right] \quad \text { for } \quad|p| \leq p_{\mathbf{F}},
$$

where $d$ is a fitting parameter, dependent on the metal under study. For momenta $p$ close to the FS, the approximation $d /\left[e-\left(p / p_{\mathrm{F}}\right)^{2}\right]$ in Eq. (2c) follows from the form of the energy denominator in the Bethe-Goldstone equation. The value of parameter $e=1.06$ in Eq. (2c), which provides the best fit of $\varepsilon_{\mathrm{c}}(p)$ to $\varepsilon_{\text {jell }}\left(p, r_{\mathrm{s}}\right)-$ $\varepsilon_{b}(p)$, appears to be universal in the whole range of electron densities, described by the parameter $r_{\mathrm{s}}$.

The corresponding (isotropic) 1D ACAR spectrum is equal to

$$
N\left(p_{z}\right)=N_{\mathrm{b}}\left(p_{z}\right)+N_{\mathrm{c}}\left(p_{z}\right)
$$

where

$$
N_{\mathrm{b}}\left(p_{z}\right)=\pi\left\{p_{\mathrm{F}}^{2}(a+b / 2+c / 3)-p_{z}^{2}\left[a+b\left(p_{z} / p_{\mathrm{F}}\right)^{2} / 2+c\left(p_{z} / p_{\mathrm{F}}\right)^{4} / 3\right]\right\}
$$




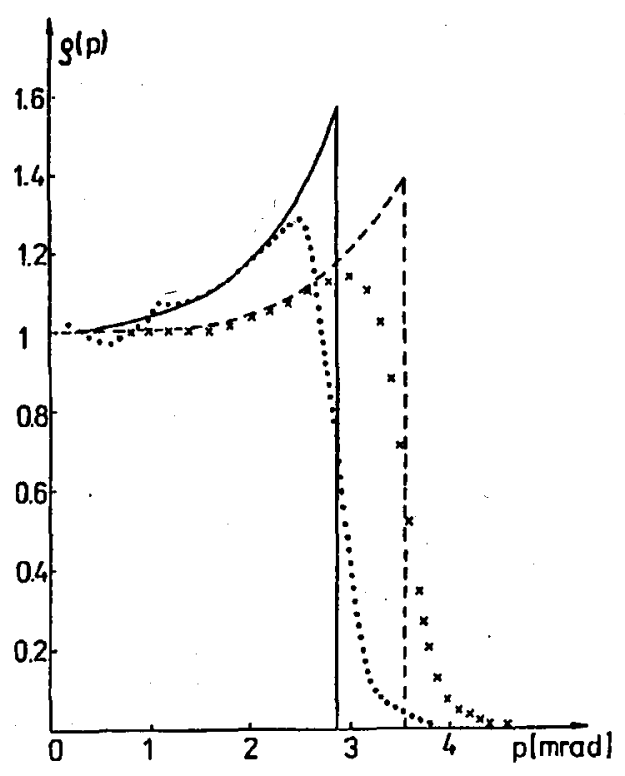

Fig. 2. Relative (isotropic) momentum densities $\rho(p) / \rho(0) \approx \varepsilon(p) / \varepsilon(0)$ in Na (broken line and crosses) and $K$ (solid line and dots). Solid and broken lines represent results of Ref. [14] obtained by fitting biparabolic form (2a) to 2D ACAR curves. Crosses and dots display reconstructed densities $\rho_{\text {rec }}(p)$.

and

$$
\begin{aligned}
N_{c}\left(p_{z}\right) & =2 \pi p_{\mathrm{F}}^{2} \int_{p_{z} / p_{\mathrm{F}}}^{1} p \varepsilon_{\mathrm{c}}\left(p / p_{\mathrm{F}}\right) \mathrm{d} p \\
= & \begin{cases}\pi d p_{\mathrm{F}}^{2} \ln (7) & \text { for } p_{z} / p_{\mathrm{F}} \leq 0.8, \\
\pi d p_{\mathrm{F}}^{2} \ln \left\{\left[1.06-\left(p_{z} / p_{\mathrm{F}}\right)^{2}\right] / 0.06\right\} & \text { for } p_{z} / p_{\mathrm{F}} \leq 0.8 .\end{cases}
\end{aligned}
$$

Values of $N_{\mathrm{c}}\left(p_{z}\right)$, for a good experimental statistics, are a few times higher than the experimental statistical error (especially for momenta close to $0.8 p_{\mathrm{F}}$ ). Parameter $d$ may be determined from the constant value of $N^{\exp }\left(p_{z}\right)-N_{\mathrm{b}}\left(p_{z}\right)$ in the region $0 \leq p \leq 0.8 p_{\mathrm{F}}$. Precise analysis of $N_{\mathrm{b}}\left(p_{z}\right)$ and $N_{\mathrm{c}}\left(p_{z}\right)$ requires, first of all, correct subtraction of core contribution. It is also important to investigate these metals in which high momentum components (HMC) do not influence visibly the shape of $N^{\exp }\left(p_{z}\right)$. It seems that $\mathrm{Na}$ is the most appropriate metal for such analysis, as seen in Fig. 2. On the other hand, in alkali metals the Fermi momentum is rather small and the influence of the resolution function of the equipment can disable us to study $\rho_{\text {rec }}(p)$ and $N^{\exp }\left(p_{z}\right)$ in the neighborhood of $0.8 p_{\mathrm{F}}$. As a conclusion, detailed studies of $N_{\mathrm{c}}\left(p_{z}\right)$ require the equipment of high resolution, as the one used by Kubica and Stewart [7]. 


\section{Methods of extracting momentum density $p(p)$ from 1D ACAR data}

Here we discuss the methods of reconstruction of momentum density $\rho(p)$ from its projections. and the ways of reducing the statistical experimental error. Alternative reconstruction techniques for $1 \mathrm{D}$ and 2D ACAR data were described by Pecora [8] as well as by Cormack [9] and Reiter and Silver [10]. We recommend techniques of Refs. [9] and [10] for the following reasons:

1) The expansion of experimental data into orthogonal polynomials has the least squares approximation properties of such functions and hence properly takes experimental errors into account.

2) The projections of $\rho(p)$, which are measured by experiment, are interdependent as they represent integrals of the same density (cf. Eq. (1)). So-called "consistency condition" for 2D data was discussed in Ref. [11] where it was shown that this condition suppresses the noise in the data.

Moreover, very few projections are necessary for this procedure (e.g. in $\mathrm{Na}$ only one projection is sufficient, cf. Fig. 2). Of course, in order to reproduce properly the shape of anisotropic density $\rho(p)$ the best way is to perform reconstruction of 2D ACAR spectra. Near the FS, however, the analysis of 1D ACAR curves seems to be more reliable.

The only quantity which can be estimated exactly from $N\left(p_{z}\right)$ is the isotropic component of the density, $\rho_{0}(p) . \rho_{0}(p)$ describes the "true" isotropic component of $\rho_{\exp }(p)$ properly, only if $N\left(p_{z}\right)$ are almost isotropic (the case of nearly isotropic FS as well as small contribution of HMC, as it takes place in $\mathrm{Na}$ in contrast to $\mathrm{K}$ ). $\rho_{0}(p)$ is related to isotropic component of $N\left(p_{z}\right), g_{0}(p)$, according to the formula [12]:

$$
\rho_{0}(p)=-\frac{1}{p} \frac{\mathrm{d} g_{0}(p)}{\mathrm{d} p} .
$$

However, calculation of derivative of $g_{0}(p)$ in Eq. (4) is troublesome because $g_{0}(p)$ is very sensitive to the experimental errors. In the case of 1D ACAR data, the expansion of $g_{0}(p)$ into the Chebyshev polynomials [13] of the first kind,

$$
g_{0}(p)=\sum_{m=0}^{N} a_{m} T_{2 m}(p)
$$

seems to be the most favorable because it allows to reduce the statistical experimental error in the highest degree. The resulting isotropic component $\rho_{0}(p)$ (Eq. (4)) is given by

$$
\rho_{0}(p)=-\frac{1}{p \pi} \sum_{m=1}^{N} m a_{m} U_{2 m-1}(p),
$$

where $U_{m}(p)$ are the Chebyshev polynomials of the second kind. This procedure enables us to reduce the experimental errors as well as to draw the shape of $\rho_{0}(p)$ with high accuracy, as seen in Fig. 2. 


\section{Conclusions}

In the interpretation of experimental ACAR data with respect to electron-positron enhancement factors, the biparabolic approximation (2a) of $\varepsilon(p)$ should be corrected near the FS. In this work the correction function $\varepsilon_{c}(p)$, dependent on the one parameter $d$ only, is proposed. The parameter $d$ is extractable from the constant value of $1 \mathrm{D}$ projection of $\varepsilon_{\mathrm{c}}(p)$ (i.e. from 1D ACAR spectrum) for $|p| \leq 0.8 p_{\mathrm{F}}$ (cf. Eq. (3c)).

The values of $b / a, c / a$ and $(b+c) / a$ extracted from experimental ACAR curves strongly depend on the way of dealing with the data and fitting procedure. Only simultaneous analysis of reconstructed momentum density $\rho_{\text {rec }}(p)$ and 1D ACAR data could enable us to determine $\varepsilon(p)$ near the FS. As the method of reconstructing $\rho(p)$ from isotropic ID ACAR data the Cormac tomography technique is recommended.

\section{Acknowledgments}

We are grateful to the authors of Ref. [14] for providing us with experimental 2D ACAR data for $\mathrm{Na}$ and $\mathrm{K}$, kindly made accessible. The work is supported by the Committee for Scientific Research, grant No. 203079101.

\section{References}

[1] G. Kontrym-Sznajd, A. Rubaszek, submitted to AIP Conf. Proc: Series, Proc. 5th Int. Workshop on Slow Positron Beam Techniques for Solids and Surfaces; Phys. Rev. $B 47$ (1993), in press.

[2] S. Daniuk, G. Kontrym-Sznajd, J. Mayers, A. Rubaszek, H. Stachowiak, P.A. Walters, R.N. West, J. Phys. F 17, 1365 (1987).

[3] H. Stachowiak, A. Rubaszek, in: Positrons at Metallic Surfaces, Ed. A. Isliii, in series Solid State Phenomena, Vol. 28-29, Trans. Tech. Publication, Aedermannsdorf 1993, p. 7 .

[4] A. Rubaszek, H. Stachowiak, J. Phys. F 15, L231 (1985).

[5] S. Kahana, Phys. Rev. 129, 1622 (1963).

[6] J. Arponen, E. Pajanne, J. Phys. F 9, 2359 (1979).

[7] P. Kubica, A.T. Stewart, Phys. Rev. Lett. 34, 852 (1975).

[8] L.M. Pecora, J. Phys., Condens. Matter 1, SA1 (1989).

[9] A.M. Cormack, J. Appl. Phys. 35, 2908 (1964); A.M. Cormack, Phys. Med. Biol. 18, 195 (1983).

[10] G. Reiter, R. Silver, Phys. Rev. Lett. 54, 1047 (1985).

[11] G. Kontrym-Sznajd, Phys. Status Solidi A 117, 227 (1990); G. Kontrym-Sznajd, in: Positron Annihilation, Mater. Sci. Forum, Vol. 105-110, Eds. Zs. Kajcsos, Cs. Szeles, Trans. Tech. Publ., Switzerland 1992, p. 325.

[12] A.T. Stewart, Can. J. Phys. 35, 168 (1957).

[13] S. Paszkowski, Numerical Application of Chebyshev Polynomials and Series, Polish Scientific Publ., Warszawa 1975 (in Polish).

[14] L. Oberli, A.A. Manuel, A.K. Singh, T. Jarlborg, L.M. Rabou, P.E. Mijnarends, T. Hyodo, A.T. Stewart, in: Positron Annihilation, Eds. P.C. Jain, R.M. Sigru, K. Gopinathan, World Scientific, Singapore 1985, p. 251. 